\title{
ANALYSIS OF THE SLOT HEATING OF THE COUPLED CAVITY LINAC CAVITY
}

\author{
Snezana Konecni and Nathan K. Bultman \\ SNS-3, Los Alamos National Laboratory \\ Los Alamos, New Mexico 87545
}

\begin{abstract}
CCL cavities are figures of revolution about the beam axis. An automated tuning program sets up the geometry for a symmetric accelerating cavity and runs SUPERFISH [1] repetitively, varying the geometry to tune each cavity to the desired frequency for the electromagnetic fields. SUPERFISH [1] solves Maxwell's equations in 2-D. A large portion of the RF power $(60-80 \%)$ applied to accelerate protons is a waste heat deposited on the inside of the copper cavity. This waste heat is removed most efficiently with water circulating through cooling passages. The waste heat needs to be removed in order to minimize thermal deformations and with it control the resonance of the cavities. A slot between the main cavity and coupled cavity receives additional heating that is not captured in the 2-D analysis. This heating causes deformation of the region and with it frequency shift. This paper covers the estimation of the slot heating and three-dimensional thermal and structural analysis of the CCL cavity.
\end{abstract}

\section{INTRODUCTION}

The Spallation Neutron Source (SNS) is an acceleratorbased facility that produces pulsed beams of neutrons by bombarding a mercury target with intense beams of 1 $\mathrm{GeV} \mathrm{H}^{-}$. Los Alamos National Laboratory is responsible for the design, fabrication, installation, and testing of the normal-conducting section of the Linear Accelerator (linac) for the SNS project. The normal-conducting linac accepts a beam from the front end system and accelerates it from $2.5 \mathrm{MeV}$ to $187 \mathrm{MeV}$. The linac consists of a drift-tube linac (DTL) up to $87 \mathrm{MeV}$ and a coupled-cavity linac (CCL) up to $188 \mathrm{MeV}$. The DTL operates at an RF resonant frequency of $402.5 \mathrm{MHz}$, while the CCL operates at $805 \mathrm{MHz}$.

CCL cavities are figures of revolution about the beam axis. An automated tuning program sets up the geometry for a symmetric accelerating cavity and runs SUPERFISH repetitively, varying the geometry to tune each cavity to the desired frequency for the electromagnetic fields. SUPERFISH solves Maxwell's equations in 2-D. The accelerator operates on an average RF duty factor of $7 \%$ an a large portion of the RF power (60-80\%) applied to accelerate the beam is waste heat deposited on the inside of the copper cavity. This waste heat is removed most efficiently with water circulating through cooling passages. The waste heat needs to be removed in order to minimize thermal deformations and with it control the resonance of the cavities.

It was decided to machine cooling channels within the copper structure on the backside of the cavity. This cooling passage design removes heat efficiently from the nose area and results in more uniform temperature and stress distributions. The current design has four concentric cooling channels with a $2 \mathrm{~mm}$ by $7.5 \mathrm{~mm}$ cross section, as shown in Figure 1.

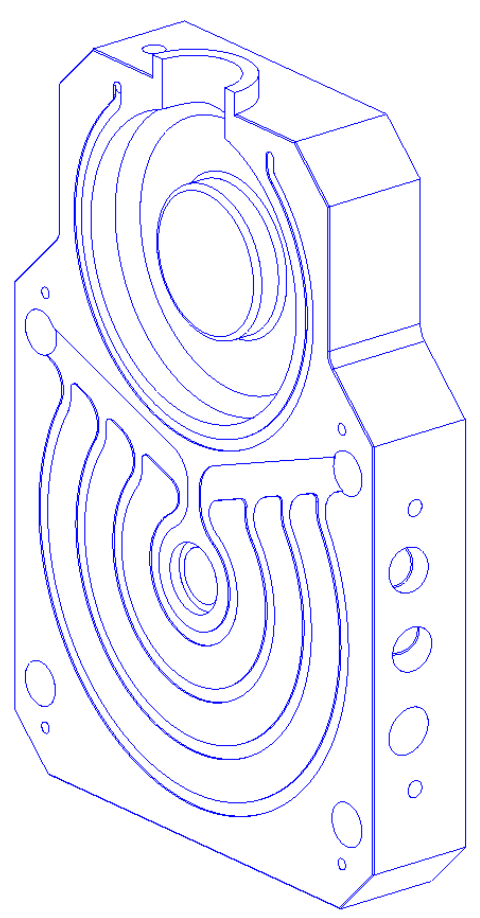

Figure 1. Back side of a CCL cavity showing the cooling channels

\section{FREQUENCY SHIFT CALCULATIONS}

The CCL is comprised of 4 modules, each of which contains 12 segments of 8 cavities each. Under normal operation about $64 \%$ of the RF power is dissipated in the CCL cavity walls. The thermal distortions caused by the RF heating result in a frequency shift as the shape and volume of the cavity change. The desired resonant frequency of the CCL cavities is $805 \mathrm{MHz}$. Importance of maintaining the required resonant frequency is to maintain 
a matched load to the RF amplifier, so that acceleration is accomplished with minimum power consumption. For any given cavity, the frequency shift can be calculated using the Slater perturbation theory. The change in frequency $d f$ is a function of the volume change of an infinitesimal volume $d V$ lying on the RF surface, as well as the electromagnetic field $H$ and the electric field $E$ for this volume. The frequency shift is given by:

$$
d f=\frac{\int\left(\mu H^{2}-\varepsilon E^{2}\right) d V}{4 U}
$$

Frequency shift can be calculated with a help of a 2-D thermal/structural analysis. Change in volume is estimated on a base of an axisymmetric model where displacement are calculated due to thermal and static pressure loadings. For the $\beta=0.549$ cavity it was estimated to be $-134 \mathrm{kHz}$.

\section{CALCULATIONS OF THE SLOT HEATING}

ANALYST [2] was used to calculate the magnetic and electric fields in the CCL cells connected to the bridge coupler. From the magnetic field plots was determined that the ratio in the magnetic field between the wall and the coupling slot is 3.18. Heat flux in the slot was calculated to be the square of the magnetic fields ratio. So for the 3-D analysis heat flux in the area of the slot was increased by 10.1 times. This resulted in power application of $25 \mathrm{~W}$ on the area of a half of a slot, $50 \mathrm{~W}$ for a full slot for $\beta=0.549$ cavity. The power load for the half cell studied is $467 \mathrm{~W}$. This power load is comparable to the output of the SUPERFISH without the $15 \%$ additional power for coupling estimated there.

\subsection{Electrical and magnetic field calculations}

3-D analysis of the electrical and magnetic field was done using ANALYST for $804 \mathrm{MHz}$. Results from this analysis are shown in Figures 2 to 4.

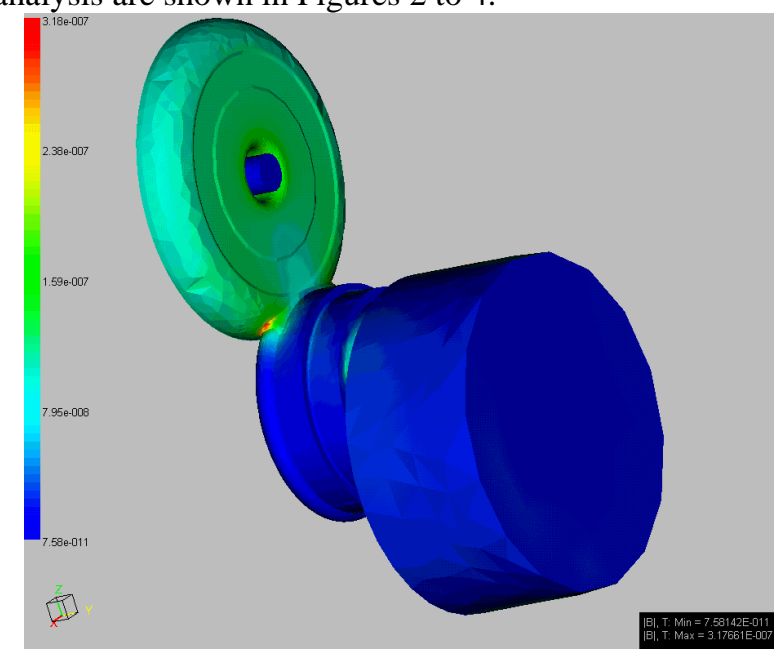

Figure 2. Magnetic field contours of the CCL cavity with the bridge coupler. The fields are normalized.

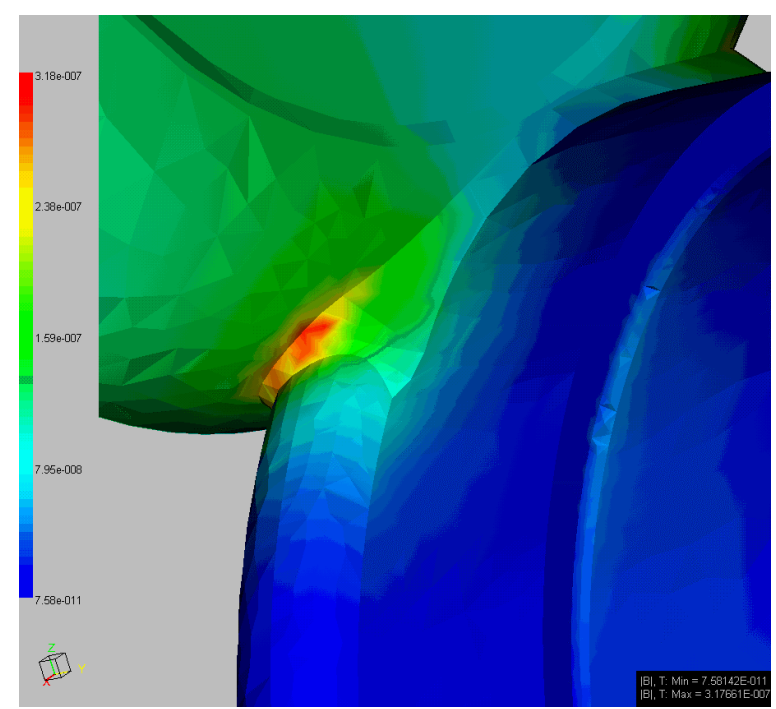

Figure 3. Detail of the magnetic field contours near the coupling cavity.

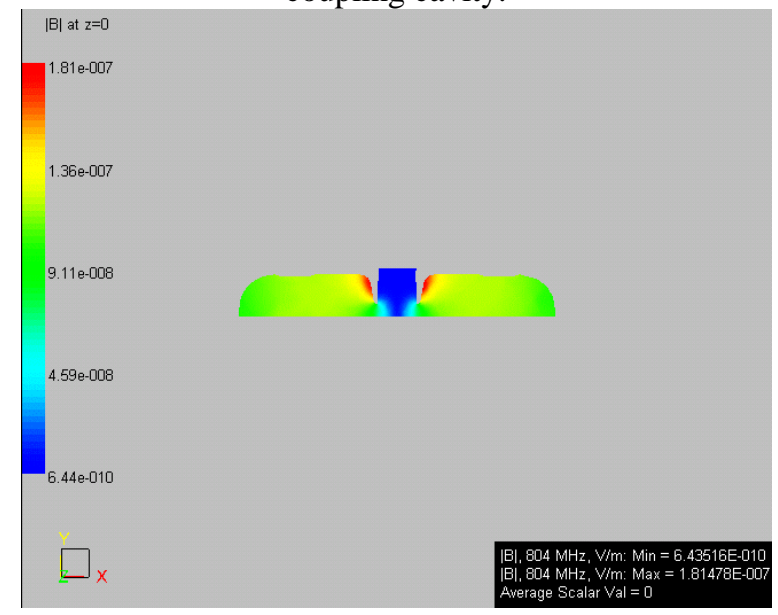

Figure 4. Magnetic field contours in the CCL cavity.

\subsection{Thermal and structural analysis}

Parameter that represents the ratio of the proton velocity and the speed of light is $\beta$. CCL cavity from the first module is at $\beta=0.4$. This is a low power cavity and the heat deposited on the inside of a half cavity is $531.8 \mathrm{~W}$. CCL cavity from the last module is at $\beta=0.549$. This is a high power cavity and the heat deposited on the inside of a half cavity is $887.17 \mathrm{~W}$.

Figure 5 shows the temperature contours obtained from 2-D analysis of $\beta=0.54$ inner cavity. Power loading for this cavity is $467 \mathrm{~W}$. Engineering factor of 1.1 was added to the heat flux. Slot heating was not considered in this analysis.

For the 3-D analysis one cavity is studied: $\beta=0.549$ (high energy cavity). Heat flux was applied in the region of the slot and its value is 10.1 larger than the heat flux found at the walls of the septum. Temperatures calculated with a 3-D analysis are shown in Figure 6. Maximum temperature is in the nose area and it is $33.93^{\circ} \mathrm{C}$, for a bulk water cooling temperature of $20^{\circ} \mathrm{C}$. 
Figure 7 shows the Von Mises stress contours. The maximum stress is $15 \mathrm{MPa}$ and is in the region of the slot. Yield strength of the copper is $34 \mathrm{MPa}$.

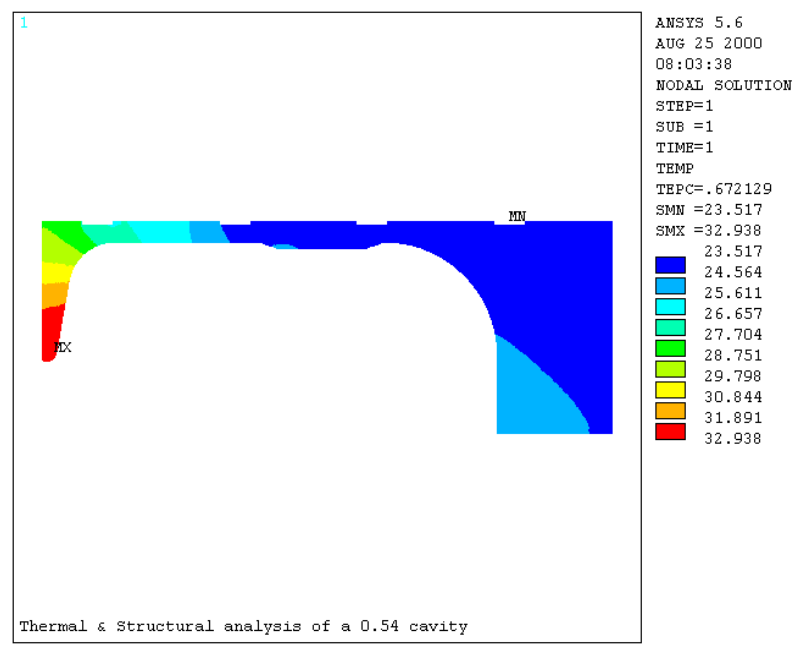

Figure 5. Temperature contours for $\beta=0.54$ inner cavity, 2-D analysis, in degrees C. Flow rate is $2.4 \mathrm{gpm}$.

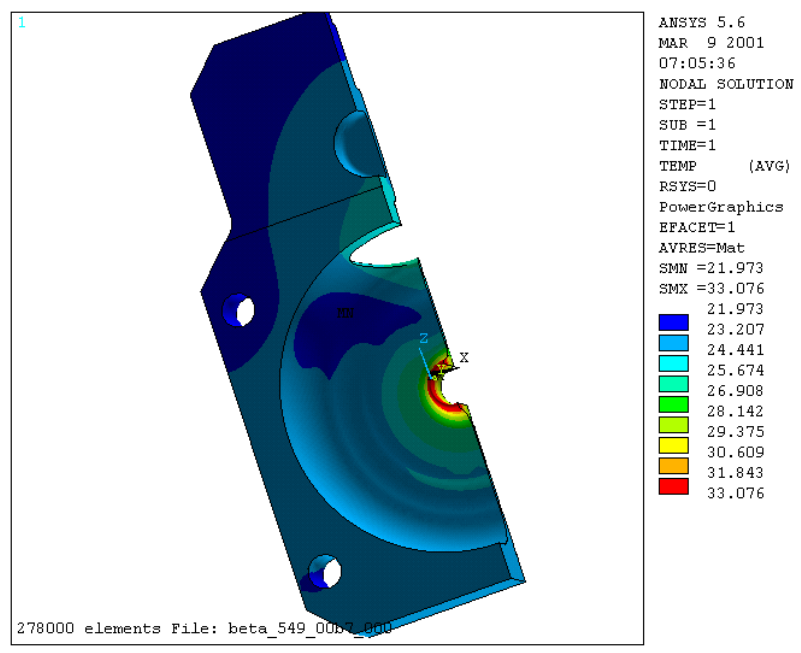

Figure 6. Temperature profile of a half CCL cavity, in degrees $\mathrm{C}$.

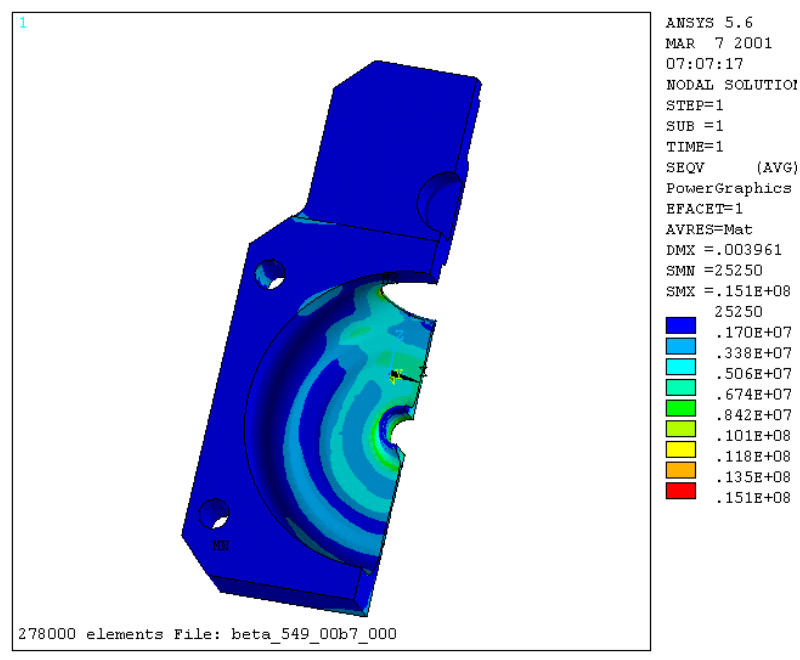

Figure 4. Von Mises stress for $\beta=0.549$ cavity. Units are $\mathrm{N} / \mathrm{m}^{2}$.

\section{CONCLUSION}

Effects of the slot heating were studied for the CCL cavities. It was estimated that approximately $50 \mathrm{~W}$ is deposited in the area of the slot due to the currents in area. This heating does not cause additional stress in the area of the slot and the current cooling scheme is adequate for the power loading at $7 \%$ duty.

\section{ACKNOWLEDGMENT}

Work supported by the Office of Basic Energy Science, Office of Science of the US Department of Energy, and by Oak Ridge National Laboratory.

\section{REFERENCES}

[1] SUPERFISH, James H. Billen and Lloyd M. Young, "POISSON SUPERFISH", LANL report LA-UR-96-1834 (revised May 2, 2001)

[2]ANALYST Accelerator, Simulation Technology and Applied Research Corporation. 